\title{
Monoclonal Antibody-Based Immunotherapy and Its Role in the Development of Cardiac Toxicity
}

\author{
Mohit Kumar ${ }^{1, *,+}$ (D) Chellappagounder Thangavel ${ }^{2,3, \dagger}$, Richard C. Becker ${ }^{1}$ and Sakthivel Sadayappan ${ }^{1}$ (D) \\ 1 Heart, Lung and Vascular Institute, Department of Internal Medicine, Division of Cardiovascular Health and \\ Disease, University of Cincinnati, Cincinnati, OH 45267, USA; beckerrc@ucmail.uc.edu (R.C.B.); \\ sadayasl@ucmail.uc.edu (S.S.) \\ 2 Department of Radiation Oncology, Sidney Kimmel Medical College, Thomas Jefferson University, \\ Philadelphia, PA 19107, USA; thangavel.chellappagounder@jefferson.edu \\ 3 Department of Dermatology, Sidney Kimmel Medical College, Thomas Jefferson University, \\ Philadelphia, PA 19107, USA \\ * Correspondence: kumarm3@ucmail.uc.edu \\ + Equally contributed.
}

Simple Summary: The application of immunotherapies to treat cancer patients has significantly improved over the last two decades and extended many patients' life spans. Monoclonal antibodies are synthetic proteins employed as immunotherapies to treat and manage cancers that require a complete understanding of cancer biology and the host's immune system. However, activated immune responses, by monoclonal antibodies, can target nonspecific cancer cells, causing frequent immune-related adverse events that can lead to permanent disorders among cancer patients. The immune-related adverse events pose a risk of cardiac toxicity that includes hypertension, heart failure, arrhythmias, and left ventricular dysfunction during and after monoclonal antibody immunotherapy. Moreover, with the mortality rate of $47 \%$ attributed to heart disease and cancer, it is imperative to employ reliable, sensitive, and clinically relevant models for efficacy and safety assessment of immune drugs for cancer and the prevention of cardiotoxicities.

Citation: Kumar, M.; Thangavel, C.; Becker, R.C.; Sadayappan, S. Monoclonal Antibody-Based Immunotherapy and Its Role in the Development of Cardiac Toxicity. Cancers 2021, 13, 86. https://doi.org/ 10.3390/cancers13010086

Received: 30 November 2020 Accepted: 26 December 2020 Published: 30 December 2020

Publisher's Note: MDPI stays neutral with regard to jurisdictional clai$\mathrm{ms}$ in published maps and institutional affiliations.

Copyright: (C) 2020 by the authors. Licensee MDPI, Basel, Switzerland. This article is an open access article distributed under the terms and conditions of the Creative Commons Attribution (CC BY) license (https:// creativecommons.org/licenses/by/ $4.0 /)$.

Abstract: Immunotherapy is one of the most effective therapeutic options for cancer patients. Five specific classes of immunotherapies, which includes cell-based chimeric antigenic receptor T-cells, checkpoint inhibitors, cancer vaccines, antibody-based targeted therapies, and oncolytic viruses. Immunotherapies can improve survival rates among cancer patients. At the same time, however, they can cause inflammation and promote adverse cardiac immune modulation and cardiac failure among some cancer patients as late as five to ten years following immunotherapy. In this review, we discuss cardiotoxicity associated with immunotherapy. We also propose using human-induced pluripotent stem cell-derived cardiomyocytes / cardiac-stromal progenitor cells and cardiac organoid cultures as innovative experimental model systems to (1) mimic clinical treatment, resulting in reproducible data, and (2) promote the identification of immunotherapy-induced biomarkers of both early and late cardiotoxicity. Finally, we introduce the integration of omics-derived highvolume data and cardiac biology as a pathway toward the discovery of new and efficient nontoxic immunotherapy.

Keywords: cardiomyocyte; heart failure; heart failure with preserved ejection fraction (HFpEF); immune checkpoint inhibitors; cardiotoxicity

\section{Introduction}

Approximately $47 \%$ of all mortality can be attributed to heart disease and cancer in the United States [1]. Traditional chemotherapies can result in cardiotoxicity, as manifested by arrhythmias and heart failure [2,3]. The emerging field of onco-immunology has led to the development of monoclonal antibodies (mAbs) to promote antitumor T-cell responses and 
response factors by inhibiting negative regulatory proteins on T-cells. This specific class of mAbs, known as immune checkpoint inhibitors (ICIs), targets cytotoxic T-lymphocyte antigen-4 (CTLA-4), programmed cell death protein-1 (PD-1), and programmed cell death ligand-1 (PD-L1). Anti-PD-1/PD-L1 mAbs are widely administered as single agents or fused with other therapeutic agents to boost T-cell function and destroy cancer cells in 50 different cancer types [4].

Immunotherapies pose a risk of cardiac toxicity, including hypertension, heart failure, QT interval prolongation, left ventricular dysfunction (LVD), and heart failure with preserved ejection fraction (HFpEF), during and after therapy [5-7], as shown in our model (Figure 1). Pathogenic cardiotoxicity is also associated with other risk factors, including age, drug dosage, and pre-existing cardiovascular disease. A clear understanding of the interaction between cancer therapies and the cardiovascular system at the molecular level can facilitate the early detection and prevention of cardiotoxicity. In this review, we discuss immunotherapy known to cause cardiovascular toxicity. We also outline novel platforms and strategies for investigating cardiovascular toxicity by employing humaninduced pluripotent stem cell-derived cardiomyocytes (hiPSC-CMs) and cardiac organoids, along with traditional biomarker detection and cardiovascular imaging.

\section{Application of immune checkpoint inhibitors (ICls)}

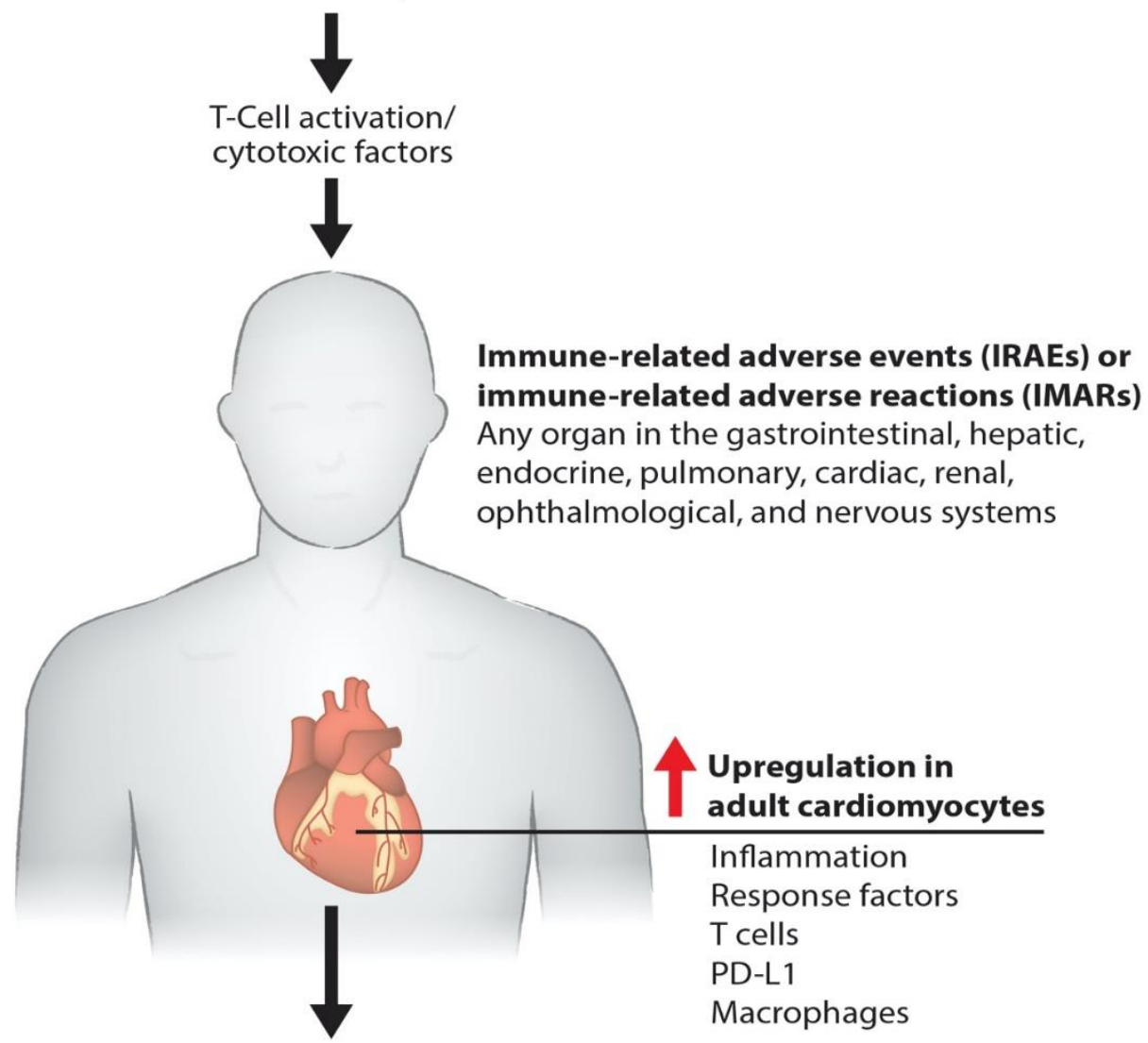

Clinical and subclinical signs/symptoms

Myocarditis/myocyte damage

Systolic and diastolic dysfunction and HFpEF

Heart failure and sudden cardiac death

Figure 1. Molecular pathogenesis of immunotherapy-induced cardiac toxicity. Schematics describe how the ICIs such as anti-PD-1, anti-PD-L1, and anti-CTLA-4 promote myocarditis via cardiac inflammation, causing myocyte damage. Cardiac myocyte dysfunction impairs systolic and diastolic function and causes failure and death. 


\section{Contemporary Cancer Immunotherapy}

Simply stated, immunotherapies are introduced to either activate or suppress the immune system. This form of treatment is the product of several decades of cancer research, together with an increased understanding of antitumor immune responses. A major advance came in the form of immune checkpoint receptor biology and its importance in determining host responses to cancer [8-10]. Several different immunotherapy strategies are currently available for patient care, such as adoptive immune cell therapies, immune modulators (ICIs, interleukins, interferons), targeted monoclonal antibodies, and cancer vaccines. Adoptive cell therapy $(\mathrm{ACT})$ is a novel and promising method involving the direct intervention of a patient's immune system to combat a broad spectrum of cancer cells $[5,11]$. For example, natural killer T-cells bind antigens on the surface of cancer cells. Several ACT immunotherapies take advantage of this natural ability and are employed in Tumor-Infiltrating Lymphocyte Therapy (TILT), Engineered T-Cell Receptor Therapy (ETCR), Chimeric Antigen Receptor T-Cell Therapy (CARTCT), and Natural Killer Cell Therapy. However, "killer" T-cells once activated and remain viable in large numbers for a sufficiently long time to mount an effective antitumor response. In TILT, naturally occurring T-cells are collected, genetically activated, and then expanded with interleukin-2. Next, cells are reinfused into the patients' tumor microenvironment (TME) to kill tumor cells. In some patients, these engineered T-cells can also be reinfused with a new T-cell receptor that allows them to target specific cancer antigens. Similarly, patients' T-cells can be infused with the synthetic chimeric antigen receptor (CAR), which helps treat patients with certain types of large B-cell lymphoma [12].

Immunomodulators use small molecules to regulate the immune system and immune response to tumorigenesis [13]. Tumors can disable regulatory immune checkpoints to shut down immune responses, protect themselves, and proliferate. In particular, tumor cells use T-cell receptors and PD-1 protein to avoid destruction by natural killer cells. In response, checkpoint inhibitors [4] can act as antibodies against immune checkpoint receptors to bind and block the tumor's inhibitory signaling downstream from CTLA-4 and PD-1, the host immune down-regulation receptors. This results in unleashing immune responses, as well as enhancing existing cytotoxic host immune response to the cancer cells (Figure 2). Cancer vaccines [14] allow the immune system to identify tumorous threats according to their specific antigens and then mount the proper response. Each patient's tumor has a unique microenvironment, or TME, that expresses its own antigens to help distinguish cancer from normal cells. These antigens can be normal proteins produced in large numbers, or they can be new proteins because of mutations and expressed exclusively by tumor cells that become the target for the directed vaccine response. 


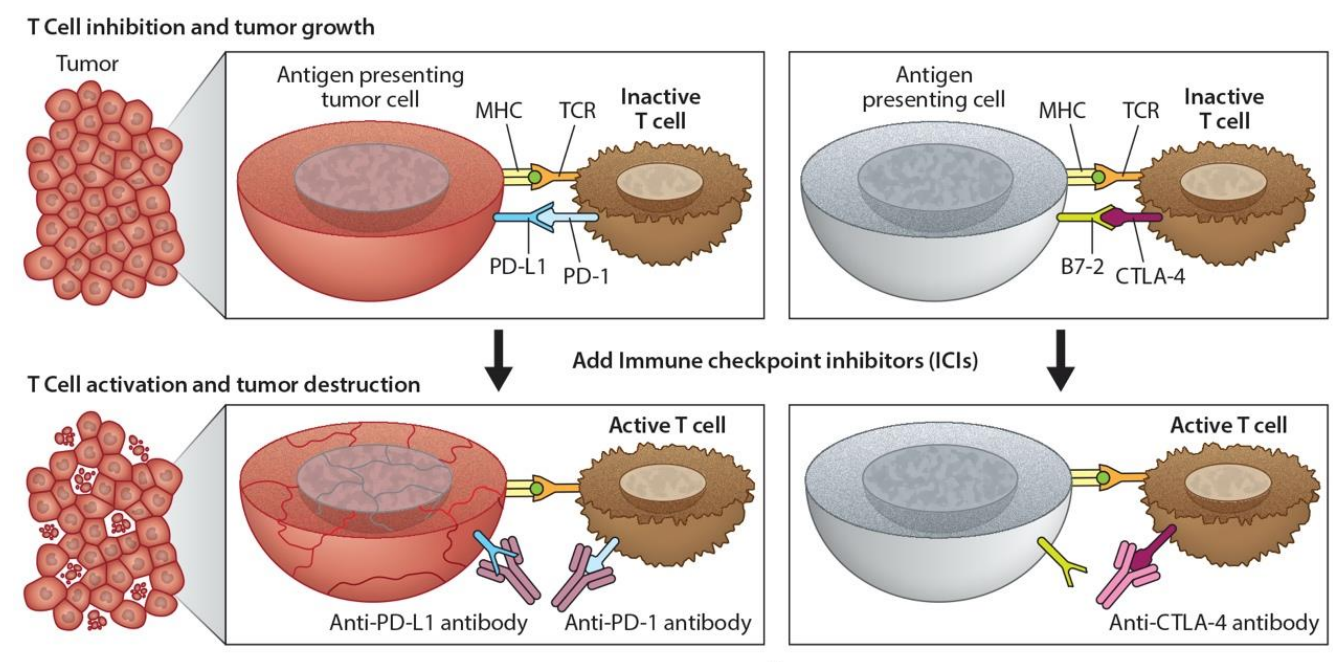

ICls injure cardimyocytes
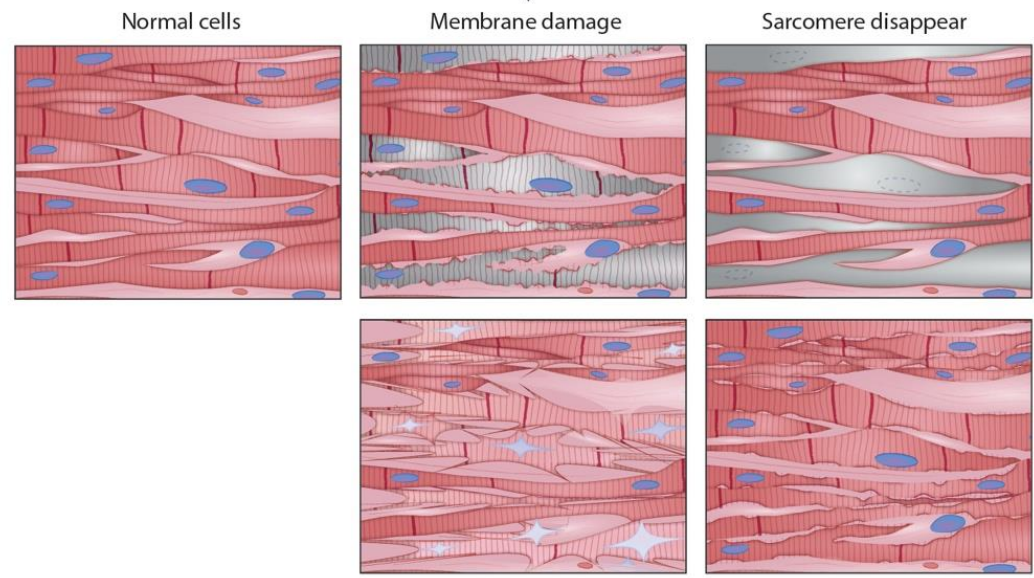

Fragmented cells

Irregular structure

Figure 2. Programmed death ligand-1 signaling and CTLA-4 deregulation facilitates tumor growth. Cartoon illustrates how PD-L1 interacts with PD-1 to inhibit T-cell activation and promote tumor cell proliferation (top panel). Working model of ICIs and promotion of cardiotoxicity (bottom panel). Cartoon illustrates how monoclonal anti-PD-1, anti-PDL-1, and anti-CTLA-4 activate T-cells following their binding to respective ligands. The activated T-cells kill/destroy the tumor cells by producing cytotoxic effects on cancer cells. In addition to tumor suppression, ICIs also promote cardiac myocyte damage, impairing function.

\section{Monoclonal Antibodies (mAbs) and Their Application in Cancer Immunotherapy}

Clinical application of mAbs immunotherapy to treat and manage many cancers has been successfully established in recent years [10]. Primary solid tumors have been effectively treated and managed with antibody-chemotherapy conjugates [10]. The development and establishment of therapeutic monoclonal antibodies require a complete understanding of tumor heterogeneity, intra-tumoral factors, protein engineering, and the interaction between cancer cells and the immune system. Researchers have now developed $\mathrm{mAbs}$ that target specific antigens in cancer cells and inhibit signals responsible for tumor growth and invasion [15]. Monoclonal antibodies are simply synthetic proteins employed as immunotherapies to treat and manage some, but not all, cancers. Monoclonal antibodies are produced in four different forms, including murine, chimeric, humanized, and human.

\subsection{Development of Therapeutic $m A$ bs}

The production of $\mathrm{mAbs}$ uses hybridoma by coupling myeloma cells with desired antibody-producing B-cells, typically from murine protein sources (-omab) [8,16]. From hybridoma, clones based on antigen specificity and immunoglobulin class are screened and 
selected to produce a single epitope. However, mAbs derived from mice are restricted owing to the generation of the innate immune response in humans. Therefore, steps were taken to generate humanized antibody by chimeric fusion of part mouse and part human proteins (-ximab), by a small portion from mouse and a major portion of protein from human (-zumab), or fully human antibody by using only human proteins (-umab) to overcome deficiencies in the mouse immune system. Around 5\% of humanized mAbs contains mouse-derived antigen-binding factor, and this portion of humanized $\mathrm{mAbs}$ is generally engineered from human sources. However, mAbs generated from hybridomas of human or humanized mouse origin systems using high-throughput immunoassays have the strongest affinities to antigens [17].

\subsection{Types of $m A b s$}

Three types of monoclonal antibodies are employed in clinics to treat cancer patients to boost their immune system includes (1) Naked mAbs, (2) Conjugated mAbs, and (3) Bispecific $m A b s$. Naked $m A b s$ are not conjugated with drug or radioactive material [18]. Conjugated $\mathrm{mAbs}$ are radiolabeled and chemolabeled antibodies [19]. Bispecific mAbs consist of parts of two different binding fragments of Abs, attaching two different proteins and bringing two cells in proximity to one another.

\subsection{Mechanism of Action of mAbs in Cancer Treatment}

Monoclonal antibodies target cancer cells in different and unique ways $[7,9,20,21]$. Naked antibodies function by themselves without any type of conjugated drug or radioactive materials. Most naked mAbs bind to antigen-bearing cancer cells, healthy cells, and free-floating proteins. Naked mAbs work by boosting the host immune system against cancer cells by binding to an antigen-presenting cancer cell. For example, alemtuzumab binds to the CD52 antigen in chronic lymphocytic leukemia patients and attracts immune cells to destroy lymphocytes. Alternatively, naked mAbs bind to immune checkpoint proteins, thereby stimulating the host immune response. The drugs pembrolizumab, Nivolumab, and Cemiplimab bind to PD-1 protein, while Atezolizumab, Avelumab, and Durvalumab bind to PD-L1 proteins to inhibit the evasion of cancer cells from host immune response. CTLA-4, also known as CD152, is a protein receptor that mediates immune checkpoint function and down-regulates immune responses. The $\mathrm{mAb}$ drug conjugate Ipilimumab binds to CTLA-4, blocking the inhibitory signal, facilitating the killing of tumor cells by cytotoxic T lymphocytes. In contrast, conjugated (tagged, labeled, or loaded) $\mathrm{mAbs}$ are combined with a chemotherapeutic drug or radioactive elements and function as a homing signal to deliver these drugs, or elements, directly to the cancer cells. An interesting example of a similar mechanism of action is the mAb drug trastuzumab that inhibits breast and stomach cancer cell proliferation by binding with HER2. An example of a radiolabeled antibody is ibritumomab tiuxetan which binds to the CD20 antigen on the surface of B-cells and delivers both drug and radioactivity to kill specific cancer cells. Another example of chemolabeled $\mathrm{mAbs}$ is brentuximab vedotin. It binds to the CD30 antigen on the surface of B-cells and attaches to a chemotherapeutic drug called monomethyl auristatin $\mathrm{E}$. The bispecific $\mathrm{mAb}$ blinatumomab binds to $\mathrm{CD} 19$ protein on lymphoma cells and CD3 protein on immune T-cells, thus triggering T-cell cytotoxicity against leukemic B-cells.

\subsection{Monoclonal Antibodies ( $m A b s$ ) in Cancer Treatment and the Development of Cardiac Toxicity}

Myocarditis is an inflammation of the heart muscle. ICIs causes myocarditis with the signs of dyspnea, fatigue, chest pain, palpitation, peripheral edema, or hypotension with elevated electrocardiogram and cardiac troponin (cTn) and sometimes no symptoms [22,23]. Similarly, ICIs can also cause inflammation of the pericardium, such as pericardial disease and pericarditis [24]. Monoclonal anti-CTLA-4 and PD-L1 are two major ICIs in immunotherapy. Monoclonal antibodies against PD-1 and PD-L1 are also an interventional therapy, as described in Table 1 and Figure 2 [25]. All immunotherapies have 
adverse side effects since activated immune responses can target nonspecific cancer cells, leading to frequent immune-related adverse events (IRAEs) or immune-related adverse reactions (IMARs) [6,26-29]. IRAEs can involve any organ in the gastrointestinal, hepatic, endocrine, pulmonary, cardiac, renal, ophthalmological, and nervous systems. IRAEs typically have delayed onset with prolonged low-grade symptoms that are, for the most part, treatable and reversible. However, some IRAEs can lead to permanent disorders among cancer patients. Monoclonal antibodies against CTLA-4, PD-1, and PD-L1 trigger IRAEs involving single organ systems, but they can simultaneously affect multiple organs in approximately $<1 \%$ of patients [2]. The most common checkpoint-inhibitor-associated IRAEs are observed in the skin (34\% of patients) with a rash over $30 \%$ of the patient's body. IRAEs can also affect the gastrointestinal tract, resulting in diarrhea or colitis occurring in $13 \%$ of cancer patients. Cardiotoxicity is rare, occurring in $0.04 \%$ to $1.14 \%$ of patients receiving immunotherapy. However, potentially fatal myocarditis and arrhythmias have been reported with a significantly higher associated mortality of $25 \%$ to $50 \%$, indicating that cardiotoxicity must be a diagnostic/prognostic consideration as these therapies expand to meet the demand $[30,31]$. A meta-analysis for lung cancer that included 22 clinical trials of ICIs showed the incidence of other cardiovascular toxicities, including pericardial disease, myocardial infarction, stroke, cardiac failure, and cardiorespiratory arrest, ranging from $0.7 \%$ to $2.0 \%$. Both cancer cells and cardiomyocytes express PD-L1, ICIs bind to cancer cells and as well as to non-target cardiomyocytes. ICIs bind to cardiomyocytes and modulates immune function, and promote muscle inflammation (autoimmune myocarditis) in heart muscle [32,33]. Additionally, ICIs can induce left ventricular hypertrophy and cause cardiac dysfunction [33-36]. However, the molecular and cellular changes during the development of cardiac dysfunction are yet to be systematically studied. Similarly, the mechanism underlying autoimmune myocarditis is not very clear. However, a shared antigen between tumor cells and cardiomyocytes could become the target for activated T-cells, leading to myocardial lymphocytic infiltration showing clinical manifestation of heart failure (HF) and cardiac conduction abnormalities [7]. PD-1 and PD-L1 are expressed in mouse and human cardiac myocytes. However, inhibiting CTLA-4 and deleting PD-l have led to autoimmune myocarditis, and several cases of HF have been reported in melanoma cancer patients treated with checkpoint inhibitors [25]. In a PD-1 knockout mouse study, an increase in autoimmune response was responsible for decreased left ventricular systolic function and wall thinning with dilated right ventricles, leading to increased mortality [17]. Moreover, diffused Immunoglobulin $\mathrm{G}$ antibody deposition was observed in cardiomyocytes of PD-1 knockout mice, suggesting an autoimmune response to the heart.

Table 1. Different ICI-induced Cardiac Toxicities in Different Cancers.

\begin{tabular}{|c|c|c|c|}
\hline Cancer Type & $\mathrm{mAb}(\mathrm{ICI})$ & Cardiac Toxicity & Reference \\
\hline Melanoma & Ipilimumab and Nivolumab & $\begin{array}{l}\text { Myositis with rhabdomyolysis, early } \\
\text { progressive cardiac electrical } \\
\text { instability and myocarditis }\end{array}$ & [37] \\
\hline Non-small cell lung cancer & Nivolumab & Massive pericardial effusion & {$[38]$} \\
\hline Hodgkin's lymphoma & Pembrolizumab (KEYTRUDA) & $\begin{array}{l}\text { Myocardial infarction, pericardial } \\
\text { effusion, pericarditis, arrhythmia and } \\
\text { cardiac tamponade }\end{array}$ & [39] \\
\hline Kidney cancer & Nivolumab & Myocarditis & {$[40]$} \\
\hline Advanced urothelial carcinoma & Pembrolizumab and Atezolizumab & Immune myocarditis & [41] \\
\hline Merkel cell carcinoma & Nivolumab & Myocarditis & {$[42]$} \\
\hline Squamous cell neck cancer & Nivolumab & $\begin{array}{c}\text { Myocarditis and ventricular } \\
\text { arrhythmia }\end{array}$ & [42] \\
\hline Squamous cell neck cancer & Pembrolizumab & Cardiac failure & {$[42]$} \\
\hline
\end{tabular}




\subsection{Cardio-Oncology: Clinical Presentation, Diagnosis, and Management of Cardiotoxicity}

Improvement in the mortality rate among cancer patients reflects better and more precise therapies. However, such therapies have also potentially increased risk factors for the cardiovascular system, resulting in long-term clinical adverse events following initial treatment. Current advancements in cardio-oncology involve highly specialized services for both cardiac health and cancer diseases. The National Institutes of Health have funded initiatives to study cardio-oncology that focus on (1) assessing cardiovascular (CV) clinical risk factors and presentation of clinical symptoms before and during cancer therapy, (2) introducing several precise cardioprotective interventions during cancer treatment, (3) balancing risk and reward as part of prescribing immunotherapy, and (4) managing post-treatment cardiotoxicity [27].

The clinical presentation of cardiotoxicity associated with immunotherapies has a spectrum of mild nonspecific symptoms to severe disease with symptoms of overt acute HF that requires inotropic support [43]. The reported clinical symptoms can be classified according to the recommendations of the American Society of Clinical Oncology clinical practice guidelines for the management of IRAEs. Life-threatening end-stage HF is the most reported contraindication in the literature, with most patients presenting a clinical syndrome of cardiogenic shock accompanied by severe conduction abnormalities, such as advanced atrioventricular blockage or ventricular tachycardia. The moderate clinical spectrum of disease ranges from chest pain, dyspnea, fatigue, peripheral edema, bilateral rales, and syncope to paroxysmal nocturnal dyspnea and palpitations $[5,44]$. Such clinical spectrum highlights the necessity of assessing CV risks in cancer patients to avoid fatal cardiotoxicity following the discontinuation of immunotherapy. It also provides a rationale for using immunosuppressive therapies to avoid such unnecessary discontinuation of effective anticancer treatments [31]. These assessments can also recognize early symptoms of hemodynamic instability that can persist during or after treatment.

Providing interventions during immunotherapy strongly depends on the diagnosis of the standard cardiac clinical manifestations as described above. Elevated serum troponin $\mathrm{T}$ levels are used to assess both prognosis and diagnosis of major adverse cardiovascular events, such as cardiovascular-related death, cardiogenic shock, cardiac arrest, or complete heart blockage as seen in myocarditis associated with immunotherapy. Similarly, along with the use of troponin, natriuretic peptides have also been proposed in screening and surveillance for higher-risk patients in the setting of immune checkpoint inhibitorrelated myocarditis. Several professional societies, including the American Society of Clinical Oncology [45], Heart Failure Association of the European Society of Cardiology [46], European Society of Medical Oncology [47], the American Society of Echocardiography, and the European Association of Cardiovascular Imaging [48], have outlined guidance for an integrated approach combining ECG, echocardiography, and biomarkers in predicting cancer immunotherapy-related cardiac failure $[49,50]$. However, all these suggestions are based on anecdotal data since the available peer-reviewed literature is limited, and clinicians have reached no consensus about the timing of cardiotoxicity onset, thus hindering accurate clinical diagnosis. Therefore, we look to more standardized patient profiling against to apply triple diagnostic scheme, e.g., before, during, and after immunotherapy, as follows: (1) definite symptoms, including abnormal echocardiography, positive biomarker and positive ECG; (2) probable clinical symptoms; and (3) possible symptoms with elevated biomarkers, but normal hemodynamic functions [2,48]. Immunosuppression is the major course of action to reduce drug-mediated cardiotoxicity with a high dose of prednisone [6]. Higher doses of steroids have not improved the outcomes of autoimmune cardiotoxicities. For stable patients in whom symptoms often appear while exercising or under stress but disappear after medication, diuretics and HF drugs should be provided until symptoms resolve. However, close monitoring needs to be done for arrhythmic patients with a low-threshold pacemaker to prevent complete heart blockage [26]. For unstable and highly symptomatic patients, immunotherapy should be stopped immediately and indefinitely until resolving IRAEs and associated cardiotoxicity [30]. Conventional cardiac 
failure therapies, such as $\beta$-blockers, calcium channel blockers, and renin-angiotensin system inhibitors, can be applied for severe and unstable patients who will show symptoms at any time, even at rest [6].

\section{Preclinical Evaluation of mAbs in the Promotion of Cardiotoxicity}

The manifestation of adverse cardiac effects in immunotherapy, both long- and shortterm, is poorly understood from a mechanistic perspective. The use of whole-animal models in vivo and in vitro studies is insufficient and does not accurately reflect early and late cardiotoxic responses of the human myocardium. Most pharmaceutical drugs targeting cancer cells are currently being withdrawn based on adverse cardiotoxicity, even after extensive testing in animal models. This calls for developing reliable, sensitive, and clinically relevant models for efficacy and safety assessment of immune drugs for cancer. Human embryonic stem cells and hiPSC-CMs have recently been applied as an in vitro research tool for modeling diseases to elucidate drug-induced pathological cardiotoxicity mechanisms at the cellular level [51]. Such models can also be predictive of adverse effects of different immunotargets at the genome and epigenome levels. Today, hiPSC-CMs can be produced in less than 2 weeks, using chemically improved cardiac differentiation methods, as well as genetically modified, using modern genome editing technologies/tools [52]. Moreover, monoclonal hiPSC-CMs can be produced on a large scale for a rigorous and reproducible model of cardiotoxicity testing in vitro and on a smaller scale for patient-specific testing. The improved protocol to generate hiPSC-CMs without ethical challenges has given hope for developing targeted therapies for cancer patients in preclinical testing and may replace, or reduce, cardiac safety assessment assays in the future [53]. Human iPSC-CMs are authentically similar to cardiomyocytes based on the expression of ion-channels and sarcomere proteins, which helped in their initial identification and characterization. Moreover, they have similar electrophysiological properties, such as action potentials and calcium transients, linked to contractions, and, in particular, regular spontaneous beating. In addition, hiPSC-CMs at the cellular levels can mimic other cardiovascular disease phenotypes, such as long-QT syndrome, cardiomyopathies, and congenital heart diseases. These hiPSC-CMs are also a suitable model for studying the response of inotropic drugs, such as norepinephrine and beating rates upon electrical stimulation. Furthermore, hiPSC-CMs can be cultured as simple 2D or 3D organoids or developed into various complex genetic compositions that resemble adult cardiac tissues [52-54]. Cancer-associated cardiotoxicity has been characterized as acute, sub-acute, and chronic. Cardiac dysfunctions, such as pericarditis or myocarditis, along with abnormal electrical conduction and cardiomyopathies, are typically manifested as sub-acute cardiotoxicity (within 1 year of therapy). However, chronic toxicities have been reported in the range of $1.6 \%$ to $23 \%$ with late-onset occurring $10-20$ years after therapy, owing to some new stress or previously injured cardiac substrate. Therefore, hiPSC-CMs are an excellent and attractive model for studying time-dependent effects of cancer immunotherapies since early cardiac markers could help detect and predict long-term cardiotoxicity [55]. However, further studies are needed to (1) determine the role of other cardiac cell types such as fibroblasts, endothelial cells, and atrial cells and (2) determine the requirement for additional systemic stimuli (hormonal, cardiotoxic metabolites) for cardiotoxicity not present in vitro. Also, to avoid false positives in new cancer immune-targeting drugs, traditional toxicity assays, or slow and deliberate dose-escalating clinical studies need to be performed along with hiPSC-CMs assays. Recently, Trastuzumab's toxic effects have been confirmed using the hiPSC-CMs platform, showing that it blocked expressed human epidermal growth factor receptor and interfered with NRG1 signaling, leading to cardiotoxicity $[56,57]$. Furthermore, Trastuzumab decreased oxidative phosphorylation and glucose use, along with mitochondrial dysfunction in hiPSC-CMs, leading to cardiac toxicity that exhibited significant cardiac impairment but no cell death [58]. 
Although the use of hiPSC-CMs for high-throughput drug screening is becoming commonplace, it is more challenging to use hiPSC-CMs for hazard identification and risk assessment during drug development. Moreover, the need has arisen for robust reproducibility of fit-to-purpose preparation of hiPSC-CMs for clinically recognized gold standards $[16,52-54,57]$. These complex human-relevant preparations need to pass rigorous testing for reproducibility and justification for the higher costs. Currently, hiPSC-CMs are promising based on their supportive role in drug-induced cardiotoxicity safety testing during clinical trials.

\section{Future Directions}

Even though the monitoring of cardiac output can now be achieved through noninvasive and invasive methodologies, such strategies are neither sufficient nor sensitive enough to discover early and late biomarkers responsible for ICI-induced cardiac toxicity. At the most basic level, iPSC-derived models are warranted to monitor cardiac function in response to monoclonal antibody-based immunotherapies (Figure 3). However, the ideal solution is to employ the $\mathrm{H} 9 \mathrm{C} 2$ cell line, iPSC-derived organoid culture, and mouse models to validate data reproducibility. Coupling of high-volume omics data and molecular assessment of cardiac function will enable the identification of early and late biomarkers pursuant to the development of personalized therapies for ICI-induced cardiac toxicity, as well as advance the emerging cardio-oncology field.

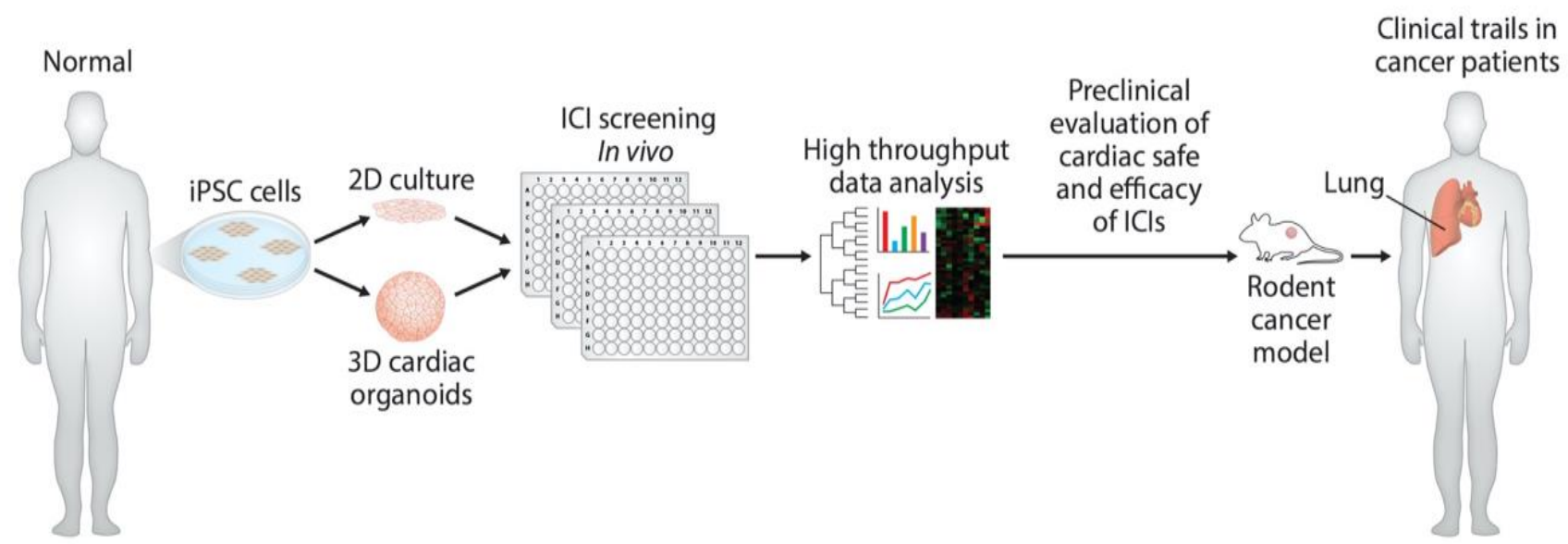

Figure 3. Development of preclinical models and determination of cardiac-safe ICIs. Diagram illustrates the use of hiPSC to develop in vitro human cardiomyocytes and cardiac organoids. These in vitro models can be used to screen monoclonal antibody-based ICIs for their cardiac toxicity. Further in vitro cardiac-safe ICIs can now be tested for toxicity in mouse tumor models and in cancer patients.

\section{Conclusions}

The manifestation of adverse cardiac effects in monoclonal antibodies immunotherapies, both long- and short-term, is poorly understood from a mechanistic perspective. Using the new platforms and strategies for investigating cardiovascular toxicity by employing human-induced pluripotent stem cell-derived cardiomyocytes (hiPSC-CMs) and cardiac organoids, along with traditional biomarker detection and cardiovascular imaging would improve the safety and efficacy of cancer immunotherapies. 
Author Contributions: Study design: M.K., C.T., S.S. \& R.C.B.; Manuscript writing and Editing: M.K., C.T., S.S. \& R.C.B.; Study Supervision: S.S. \& R.C.B. All authors have read and agreed to the published version of the manuscript.

Funding: This research received no external funding. The current research was funded by Heart, Lung, and Vascular Institute startup funding at the College of Medicine, University of Cincinnati. The author's funding is mentioned below in acknowledgments.

Data Availability Statement: All the data presented in this study are included in this article.

Acknowledgments: Kumar was supported by the American Heart Association Predoctoral Fellowship (17PRE33630192). Sadayappan has received support from National Institutes of Health grants (R01 HL130356, R01 HL105826, R01 AR067279, R01 AR078001 and RO1/R56 HL139680), American Heart Association, Cardiovascular Genome-Phenome Study (15CVGPSD27020012), Catalyst (17CCRG33671128), Institutional Undergraduate Student (19UFEL34380251) and Transformation (19TPA34830084) awards, the Phospholamban (PLN) Foundation (PLN crazy idea) awards, as well as AstraZeneca, MyoKardia, Merck, and Amgen.

Conflicts of Interest: Becker serves on scientific advisory boards for Janssen and Basking Biosciences and DSMB Committees for Ionis Pharmaceuticals, Akcea Therapeutics and Novartis. Sadayappan provided consulting and collaborative research studies to the Leducq Foundation, Red Saree Inc., Greater Cincinnati Tamil Sangam, MyoKardia, Merck and Amgen, but such work is unrelated to the content of this article. No other disclosures are reported.

$\begin{array}{ll}\text { Abbreviations } & \\ \text { ACT } & \text { Adoptive cell therapy } \\ \text { TIL } & \text { Tumor-Infiltrating Lymphocyte } \\ \text { TCR } & \text { T-Cell Receptor } \\ \text { CAR } & \text { Chimeric Antigen Receptor } \\ \text { hiPSC-CMs } & \text { human-induced pluripotent stem cell-derived cardiomyocytes } \\ \text { CTLA-4 } & \text { Cytotoxic T-lymphocyte-associated antigen 4 } \\ \text { ASE } & \text { American Society of Echocardiography } \\ \text { EACVI } & \text { European Association of Cardiovascular Imaging } \\ \text { IRAEs } & \text { Immune-related adverse events } \\ \text { IMARs } & \text { Immune-related adverse reactions } \\ \text { ICI } & \text { Immune checkpoint inhibitors } \\ \text { LVD } & \text { Left ventricular dysfunction } \\ \text { HFpEF } & \text { Heart failure with preserved ejection fraction } \\ \text { PD-1 } & \text { Programmed cell death protein-1 } \\ \text { PD-L1 } & \text { Programmed death ligand-1 }\end{array}$

\section{References}

1. Lenneman, C.G.; Kimmick, G.G.; Sawyer, D.B. Epidemiology of Cardio-Oncology. In Cardio-Oncology: The Clinical Overlap of Cancer and Heart Disease; Kimmick, G.G., Lenihan, D.J., Sawyer, D.B., Mayer, E.L., Hershman, D.L., Eds.; Springer: Cham, Switzerland, 2017; pp. 1-14.

2. Palaskas, N.; Lopez-Mattei, J.; Durand, J.B.; Iliescu, C.; Deswal, A. Immune Checkpoint Inhibitor Myocarditis: Pathophysiological Characteristics, Diagnosis, and Treatment. J. Am. Heart Assoc. 2020, 9, e013757. [CrossRef]

3. Zarifa, A.; Lopez-Mattei, J.; Palaskas, N.; Iliescu, C.; Durand, J.B.; Kim, P.Y. Immune Checkpoint Inhibitors (ICIs)-Related Cardiotoxicity. Adv. Exp. Med. Biol. 2020, 1244, 277-285.

4. Robert, C. A decade of immune-checkpoint inhibitors in cancer therapy. Nat. Commun. 2020, 11, 3801. [CrossRef]

5. Dong, J.; Chen, H. Cardiotoxicity of Anticancer Therapeutics. Front. Cardiovasc. Med. 2018, 5, 9. [CrossRef]

6. Jain, V.; Bahia, J.; Mohebtash, M.; Barac, A. Cardiovascular Complications Associated with Novel Cancer Immunotherapies. Curr. Treat. Options Cardiovasc. Med. 2017, 19, 36. [CrossRef]

7. Said, R.; Nickolich, M.; Lenihan, D.J.; Tsimberidou, A.M. Cardiotoxicity of Anticancer Therapies. In Cardio-Oncology: The Clinical Overlap of Cancer and Heart Disease; Kimmick, G.G., Lenihan, D.J., Sawyer, D.B., Mayer, E.L., Hershman, D.L., Eds.; Springer: Cham, Switzerland, 2017; pp. 15-42.

8. Berger, M.; Shankar, V.; Vafai, A. Therapeutic applications of monoclonal antibodies. Am. J. Med. Sci. 2002, 324, 14-30. [CrossRef]

9. Hafeez, U.; Gan, H.K.; Scott, A.M. Monoclonal antibodies as immunomodulatory therapy against cancer and autoimmune diseases. Curr. Opin. Pharmacol. 2018, 41, 114-121. [CrossRef]

10. Scott, A.M.; Wolchok, J.D.; Old, L.J. Antibody therapy of cancer. Nat. Rev. Cancer 2012, 12, 278-287. [CrossRef] 
11. Rohaan, M.W.; Wilgenhof, S.; Haanen, J. Adoptive cellular therapies: The current landscape. Virchows Arch. Int. J. Pathol. 2019, 474, 449-461. [CrossRef]

12. Feins, S.; Kong, W.; Williams, E.F.; Milone, M.C.; Fraietta, J.A. An introduction to chimeric antigen receptor (CAR) T-cell immunotherapy for human cancer. Am. J. Hematol. 2019, 94, S3-S9. [CrossRef]

13. Stanculeanu, D.L.; Daniela, Z.; Lazescu, A.; Bunghez, R.; Anghel, R. Development of new immunotherapy treatments in different cancer types. J. Med. Life 2016, 9, 240-248.

14. Hollingsworth, R.E.; Jansen, K. Turning the corner on therapeutic cancer vaccines. NPJ Vaccines 2019, 4, 7. [CrossRef]

15. Zhang, X.; Soori, G.; Dobleman, T.J.; Xiao, G.G. The application of monoclonal antibodies in cancer diagnosis. Exp. Rev. Mol. Diagn. 2014, 14, 97-106. [CrossRef]

16. Lu, R.M.; Hwang, Y.C.; Liu, I.J.; Lee, C.C.; Tsai, H.Z.; Li, H.J.; Wu, H.C. Development of therapeutic antibodies for the treatment of diseases. J. Biomed. Sci. 2020, 27, 1. [CrossRef]

17. Chen, Y.; Hu, Z.; Wang, Q.; Ge, Y.; Bai, L.; Wang, X.; Zhang, X. Generation and characterization of four novel monoclonal antibodies against human programmed death-1 molecule. Hybridoma 2010, 29, 153-160. [CrossRef]

18. Wei, G.; Wang, J.; Huang, H.; Zhao, Y. Novel immunotherapies for adult patients with B-lineage acute lymphoblastic leukemia. J. Hematol. Oncol. 2017, 10, 150. [CrossRef]

19. Jin, J.; Park, G.; Park, J.B.; Kim, S.; Kim, H.; Chung, J. An anti-EGFR x cotinine bispecific antibody complexed with cotinineconjugated duocarmycin inhibits growth of EGFR-positive cancer cells with KRAS mutations. Exp. Mol. Med. 2018, 50, 1-14. [CrossRef]

20. Marhelava, K.; Pilch, Z.; Bajor, M.; Graczyk-Jarzynka, A.; Zagozdzon, R. Targeting Negative and Positive Immune Checkpoints with Monoclonal Antibodies in Therapy of Cancer. Cancers 2019, 11, 1756. [CrossRef]

21. Pento, J.T. Monoclonal Antibodies for the Treatment of Cancer. Anticancer Res. 2017, 37, 5935-5939.

22. Spallarossa, P.; Tini, G.; Sarocchi, M.; Arboscello, E.; Grossi, F.; Queirolo, P.; Zoppoli, G.; Ameri, P. Identification and Management of Immune Checkpoint Inhibitor-Related Myocarditis: Use Troponin Wisely. J. Clin. Oncol. Off. J. Am. Soc. Clin. Oncol. 2019, 37, 2201-2205. [CrossRef]

23. Wang, D.Y.; Salem, J.E.; Cohen, J.V.; Chandra, S.; Menzer, C.; Ye, F.; Zhao, S.; Das, S.; Beckermann, K.E.; Ha, L.; et al. Fatal Toxic Effects Associated With Immune Checkpoint Inhibitors: A Systematic Review and Meta-analysis. JAMA Oncol. 2018, 4, 1721-1728. [CrossRef]

24. Imazio, M.; Gaita, F.; LeWinter, M. Evaluation and Treatment of Pericarditis: A Systematic Review. JAMA 2015, 314, 1498-1506. [CrossRef]

25. Varricchi, G.; Marone, G.; Mercurio, V.; Galdiero, M.R.; Bonaduce, D.; Tocchetti, C.G. Immune Checkpoint Inhibitors and Cardiac Toxicity: An Emerging Issue. Curr. Med. Chem. 2018, 25, 1327-1339. [CrossRef]

26. Castrillon, J.A.; Eng, C.; Cheng, F. Pharmacogenomics for Immunotherapy and Immune-related Cardiotoxicity. Hum. Mol. Genet. 2020, 29, R186-R196. [CrossRef]

27. Minasian, L.; Dimond, E.; Davis, M.; Adhikari, B.; Fagerstrom, R.; Fabian, C.; Floyd, J.; Unger, J.M.; Douglas, P.S.; Mustian, K.M.; et al. The Evolving Design of NIH-Funded Cardio-Oncology Studies to Address Cancer Treatment-Related Cardiovascular Toxicity. JACC Cardio Oncol. 2019, 1, 105-113. [CrossRef]

28. Zaidi, N.; Jaffee, E.M. Immunotherapy transforms cancer treatment. J. Clin. Investig. 2019, 129, 46-47. [CrossRef]

29. Saiki, H.; Petersen, I.A.; Scott, C.G.; Bailey, K.R.; Dunlay, S.M.; Finley, R.R.; Ruddy, K.J.; Yan, E.; Redfield, M.M. Risk of Heart Failure with Preserved Ejection Fraction in Older Women After Contemporary Radiotherapy for Breast Cancer. Circulation 2017, 135, 1388-1396. [CrossRef]

30. Upadhrasta, S.; Elias, H.; Patel, K.; Zheng, L. Managing cardiotoxicity associated with immune checkpoint inhibitors. Chronic Dis. Transl. Med. 2019, 5, 6-14. [CrossRef]

31. Ryan, T.D.; Nagarajan, R.; Godown, J. Pediatric Cardio-Oncology: Development of Cancer Treatment-Related Cardiotoxicity and the Therapeutic Approach to Affected Patients. Curr. Treat. Options Oncol. 2019, 20, 56. [CrossRef]

32. Jain, V.; Mohebtash, M.; Rodrigo, M.E.; Ruiz, G.; Atkins, M.B.; Barac, A. Autoimmune Myocarditis Caused by Immune Checkpoint Inhibitors Treated With Antithymocyte Globulin. J. Immunother. 2018, 41, 332-335. [CrossRef]

33. Tajmir-Riahi, A.; Bergmann, T.; Schmid, M.; Agaimy, A.; Schuler, G.; Heinzerling, L. Life-threatening Autoimmune Cardiomyopathy Reproducibly Induced in a Patient by Checkpoint Inhibitor Therapy. J. Immunother. 2018, 41, 35-38. [CrossRef]

34. Xia, W.; Chen, H.; Chen, D.; Ye, Y.; Xie, C.; Hou, M. PD-1 inhibitor inducing exosomal miR-34a-5p expression mediates the cross talk between cardiomyocyte and macrophage in immune checkpoint inhibitor-related cardiac dysfunction. J. Immunother. Cancer 2020, 8, e001293. [CrossRef]

35. Agrawal, N.; Khunger, A.; Vachhani, P.; Colvin, T.A.; Hattoum, A.; Spangenthal, E.; Curtis, A.B.; Dy, G.K.; Ernstoff, M.S.; Puzanov, I. Cardiac Toxicity Associated with Immune Checkpoint Inhibitors: Case Series and Review of the Literature. Case Rep. Oncol. 2019, 12, 260-276. [CrossRef]

36. Wang, H.; Kwak, D.; Fassett, J.; Hou, L.; Xu, X.; Burbach, B.J.; Thenappan, T.; Xu, Y.; Ge, J.B.; Shimizu, Y.; et al. CD28/B7 Deficiency Attenuates Systolic Overload-Induced Congestive Heart Failure, Myocardial and Pulmonary Inflammation, and Activated T Cell Accumulation in the Heart and Lungs. Hypertension 2016, 68, 688-696. [CrossRef]

37. Johnson, D.B.; Balko, J.M.; Compton, M.L.; Chalkias, S.; Gorham, J.; Xu, Y.; Hicks, M.; Puzanov, I.; Alexander, M.R.; Bloomer, T.L.; et al. Fulminant Myocarditis with Combination Immune Checkpoint Blockade. N. Engl. J. Med. 2016, 375, 1749-1755. [CrossRef] 
38. Saade, A.; Mansuet-Lupo, A.; Arrondeau, J.; Thibault, C.; Mirabel, M.; Goldwasser, F.; Oudard, S.; Weiss, L. Pericardial effusion under nivolumab: Case-reports and review of the literature. J. Immunother. Cancer 2019, 7, 266. [CrossRef]

39. Chen, D.Y.; Huang, W.K.; Chien-Chia Wu, V.; Chang, W.C.; Chen, J.S.; Chuang, C.K.; Chu, P.H. Cardiovascular toxicity of immune checkpoint inhibitors in cancer patients: A review when cardiology meets immuno-oncology. J. Formos Med. Assoc. 2020, 119, 1461-1475. [CrossRef]

40. Zhou, Y.W.; Zhu, Y.J.; Wang, M.N.; Xie, Y.; Chen, C.Y.; Zhang, T.; Xia, F.; Ding, Z.Y.; Liu, J.Y. Immune Checkpoint InhibitorAssociated Cardiotoxicity: Current Understanding on Its Mechanism, Diagnosis and Management. Front. Pharmacol. 2019, 10, 1350. [CrossRef]

41. Tocchetti, C.G.; Cadeddu, C.; Di Lisi, D.; Femmino, S.; Madonna, R.; Mele, D.; Monte, I.; Novo, G.; Penna, C.; Pepe, A.; et al. From Molecular Mechanisms to Clinical Management of Antineoplastic Drug-Induced Cardiovascular Toxicity: A Translational Overview. Antioxid. Redox Signal. 2019, 30, 2110-2153. [CrossRef]

42. Lyon, A.R.; Yousaf, N.; Battisti, N.M.L.; Moslehi, J.; Larkin, J. Immune checkpoint inhibitors and cardiovascular toxicity. Lancet Oncol. 2018, 19, e447-e458. [CrossRef]

43. Khouri, M.G.; Klem, I.; Shenoy, C.; Sulpher, J.; Dent, S.F. Screening and Monitoring for Cardiotoxicity During Cancer Treatment. In Cardio-Oncology: Clinical Overlap of Cancer and Heart Disease; Kimmick, G.G., Lenihan, D.J., Sawyer, D.B., Mayer, E.L., Hershman, D.L., Eds.; Springer: Cham, Switzerland, 2017; pp. 43-80.

44. Cheng, R.K.; Kirkpatrick, J.N.; Sorror, M.L.; Barac, A. Cardio-Oncology and the Intersection of Cancer and Cardiotoxicity. JACC Cardio Oncol. 2019, 1, 314-317. [CrossRef]

45. Brahmer, J.R.; Lacchetti, C.; Schneider, B.J.; Atkins, M.B.; Brassil, K.J.; Caterino, J.M.; Chau, I.; Ernstoff, M.S.; Gardner, J.M.; Ginex, P.; et al. Management of Immune-Related Adverse Events in Patients Treated With Immune Checkpoint Inhibitor Therapy: American Society of Clinical Oncology Clinical Practice Guideline. J. Clin. Oncol. Off. J. Am. Soc. Clin. Oncol. 2018, 36, 1714-1768. [CrossRef]

46. Zamorano, J.L.; Lancellotti, P.; Rodriguez Muñoz, D.; Aboyans, V.; Asteggiano, R.; Galderisi, M.; Habib, G.; Lenihan, D.J.; Lip, G.Y.H.; Lyon, A.R.; et al. 2016 ESC Position Paper on cancer treatments and cardiovascular toxicity developed under the auspices of the ESC Committee for Practice Guidelines: The Task Force for cancer treatments and cardiovascular toxicity of the European Society of Cardiology (ESC). Eur. Heart J. 2016, 37, 2768-2801. [CrossRef]

47. Curigliano, G.; Cardinale, D.; Suter, T.; Plataniotis, G.; de Azambuja, E.; Sandri, M.T.; Criscitiello, C.; Goldhirsch, A.; Cipolla, C.; Roila, F. Cardiovascular toxicity induced by chemotherapy, targeted agents and radiotherapy: ESMO Clinical Practice Guidelines. Ann. Oncol. 2012, 23, vii155-vii166. [CrossRef]

48. Gilstrap, L.; Harrison, M.; Kimmick, G.G.; Nohria, A. Management of Chemotherapy-Associated Cardiomyopathy. In CardioOncology: The Clinical Overlap of Cancer and Heart Disease; Kimmick, G.G., Lenihan, D.J., Sawyer, D.B., Mayer, E.L., Hershman, D.L., Eds.; Springer: Cham, Switzerland, 2017; pp. 81-104.

49. Curigliano, G.; Lenihan, D.; Fradley, M.; Ganatra, S.; Barac, A.; Blaes, A.; Herrmann, J.; Porter, C.; Lyon, A.R.; Lancellotti, P.; et al. Management of cardiac disease in cancer patients throughout oncological treatment: ESMO consensus recommendations. Ann. Oncol. 2020, 31, 171-190. [CrossRef]

50. Teske, A.J.; Linschoten, M.; Kamphuis, J.A.M.; Naaktgeboren, W.R.; Leiner, T.; van der Wall, E.; Kuball, J.; van Rhenen, A.; Doevendans, P.A.; Cramer, M.J.; et al. Cardio-oncology: An overview on outpatient management and future developments. Neth. Heart J. 2018, 26, 521-532. [CrossRef]

51. Sachinidis, A. Cardiotoxicity and Heart Failure: Lessons from Human-Induced Pluripotent Stem Cell-Derived Cardiomyocytes and Anticancer Drugs. Cells 2020, 9, 1001. [CrossRef]

52. Sayed, N.; Ameen, M.; Wu, J.C. Personalized medicine in cardio-oncology: The role of induced pluripotent stem cell. Cardiovasc. Res. 2019, 115, 949-959. [CrossRef]

53. Gintant, G.; Burridge, P.; Gepstein, L.; Harding, S.; Herron, T.; Hong, C.; Jalife, J.; Wu, J.C. Use of Human Induced Pluripotent Stem Cell-Derived Cardiomyocytes in Preclinical Cancer Drug Cardiotoxicity Testing: A Scientific Statement From the American Heart Association. Circ. Res. 2019, 125, e75-e92. [CrossRef]

54. Sharma, A.; McKeithan, W.L.; Serrano, R.; Kitani, T.; Burridge, P.W.; Del Alamo, J.C.; Mercola, M.; Wu, J.C. Use of human induced pluripotent stem cell-derived cardiomyocytes to assess drug cardiotoxicity. Nat. Protoc. 2018, 13, 3018-3041. [CrossRef]

55. Pinheiro, E.A.; Fetterman, K.A.; Burridge, P.W. hiPSCs in cardio-oncology: Deciphering the genomics. Cardiovasc. Res. 2019, 115, 935-948. [CrossRef]

56. Niepel, M.; Hafner, M.; Duan, Q.; Wang, Z.; Paull, E.O.; Chung, M.; Lu, X.; Stuart, J.M.; Golub, T.R.; Subramanian, A.; et al. Common and cell-type specific responses to anti-cancer drugs revealed by high throughput transcript profiling. Nat. Commun. 2017, 8, 1186. [CrossRef]

57. Kitani, T.; Ong, S.-G.; Lam, C.K.; Rhee, J.-W.; Zhang, J.Z.; Oikonomopoulos, A.; Ma, N.; Tian, L.; Lee, J.; Telli, M.L.; et al. Human-Induced Pluripotent Stem Cell Model of Trastuzumab-Induced Cardiac Dysfunction in Patients With Breast Cancer. Circulation 2019, 139, 2451-2465. [CrossRef] [PubMed]

58. Necela, B.M.; Axenfeld, B.C.; Serie, D.J.; Kachergus, J.M.; Perez, E.A.; Thompson, E.A.; Norton, N. The antineoplastic drug, trastuzumab, dysregulates metabolism in iPSC-derived cardiomyocytes. Clin. Transl. Med. 2017, 6, 5. [CrossRef] [PubMed] 